\title{
Association of High w-6/w-3 Ratio Diet with an Ophthalmic Emergency in Patient with Polymyalgia Rheumatica and Giant Cell Arteritis
}

\author{
Z. Macejová ${ }^{1, *}$ and R.B. Singh ${ }^{2}$ \\ ${ }^{1} 3^{\text {rd }}$ Internal Clinic, Faculty of Medicine, Safarik University, Kosice, Slovakia \\ ${ }^{2}$ Department of Internal Medicine and Cardiology, Halberg Hospital and Research Institute, Civil Lines, Moradabad-10, \\ UP 244001, India; TsimTsoum Institute Krakow, Poland
}

\begin{abstract}
This is a report of two patients, one having polymyalgia rheumatica (PMR) and another with giant cell arteritis (GCA). Both the patients had raised erythrocyte sedimentation rate (ESR) and C-reactive protein and were consuming high w-6/w-3 fatty acid ratio diet. Both responded to prednisolone. Low w-3 ratio diet or fish oil should be considered for prevention of recurrence.
\end{abstract}

Keywords: Inflammation, diet, joint pain, w-3 fatty acids.

\section{INTRODUCTION}

Polymyalgia rheumatica and giant cell arteritis are closely related disorders that affect elderly people and are seldom diagnosed below the age of 50 years [1-7]. These are inflammatory problems and frequently occur together. Both are syndromes of unknown cause, but genetic and enviromental factors might have a role in their pathogenesis [17]. Giant cell arteritis is a vasculitis affecting branches of the external and internal carotid arteries (Fig. 1). Headache is the most common symptom, but scalp tenderness, jaw claudication, cerebrovascular accident, aortic arch syndrome, thoracic aorta aneurysm, and dissection may also occur. Vision involvment may be serious. The symptoms of polymyalgia rheumatica seem to be related to synovitis of proximal joints and extra-articular structures. Both problems are related to chronic diseases of affluence, therefore increases in w- $6 / \mathrm{w}-3$ ratio in the diet may be associated with proinflammatory effects predisposing to these problems.

Wild - whole grains rich in w-3 fatty acids, antioxidants, vitamins and minerals may provide better protective effects compared to whole grain grown by modern farming [8-13]. Prior to the Agricultural Revolution, our diet was based on wild plants, eggs, fish and meat from wild animals, whereas today, the greatest percentage of the food supply is contributed by cereal grains, (mainly refined), refined carbohydrates and meat from farm sources [13]. These foods are rich in w6 fatty acids and low in w-3 fatty acids, fiber, phytoestrogens, minerals and antioxidants. Cardiovascular disease (CVD), diabetes mellitus, cancer, autoimmune diseases, rheumatoid arthritis, asthma, polymyalgia rheumatica, giant cell arteritis and depression may be associated with increased production of thromboxane A2, leucotrienes, interleukins-1 and 6, tumor necrosis factor-alpha and C-reactive protein [816]. Increased dietary intake of w-6 fatty acids is known to

*Address correspondence to this author at the 3-rd internal clinic, Faculty of Medicine, safarik University, Kosice, Slovakia; Tel: 0042155 6403423; Fax: 00421556403413 ;

E-mails: macejova@hotmail.com, zelmira.macejova@upjs.sk enhance the risk of all these diseases by having adverse proinflammatry effects [8-13]. There is a marked increase in the consumption of oils of sunflower, soyabean, corn and safflower in the last 4 decades with the availability of foods manufactured with these oils [13-16]. These oils are rich in w-6 fatty acids, resulting in a marked increase in the w- $6 / \mathrm{w} 3$ ratio of the diet in both developed and developing countries of the world [8-13]. It is possible that a high $\mathrm{w}-6 / \mathrm{w}-3$ status may be the cause of an increased death rate due to noncommunicable diseases [13-16]. We report two patients who were receiving a high $\mathrm{w}-6 / \mathrm{w}-3$ ratio diet in association with PMR and GCA. Dietary intakes were assessed from the weekly diet diaries filled by both the patients, during the last one week, with the help of questionnaires filled by the dietitian. The dietitian asked proving questions and used food models, food portions and food measures to find out exact quantity of foods consumed by the patient. However, such assessments of fatty acid intake are open to bias, but these are standard procedures and accepted by most investigators [14-16].

\section{CASE REPORT 1}

Patient - An 85 year- old white female was admitted to the hospital because of intensive headache, low grade fever, weight loss, pain and stiffness of the neck, shoulder and sudden visual loss. The family history revealed that a younger sister was treated for discoid lupus erythematosus, a daughter was treated for epilepsy who had positive ANA antibodies with no clinical correlation. The patient's previous medical history was unremarkable, except for ischemic heart disease.

On admission, the patient appeared confused with an intensive headache, low grade fever and pain with stiffness of the neck, sudden visual loss in the right eye and vertigo. Her blood pressure was $115 / 80 \mathrm{~mm} \mathrm{Hg}$. She had pain with stiffness of the muscles in the shoulder region and neck bilaterally and symmetrical arthralgia of the knees. The patient suffered from headaches and pain localized to the temple. 


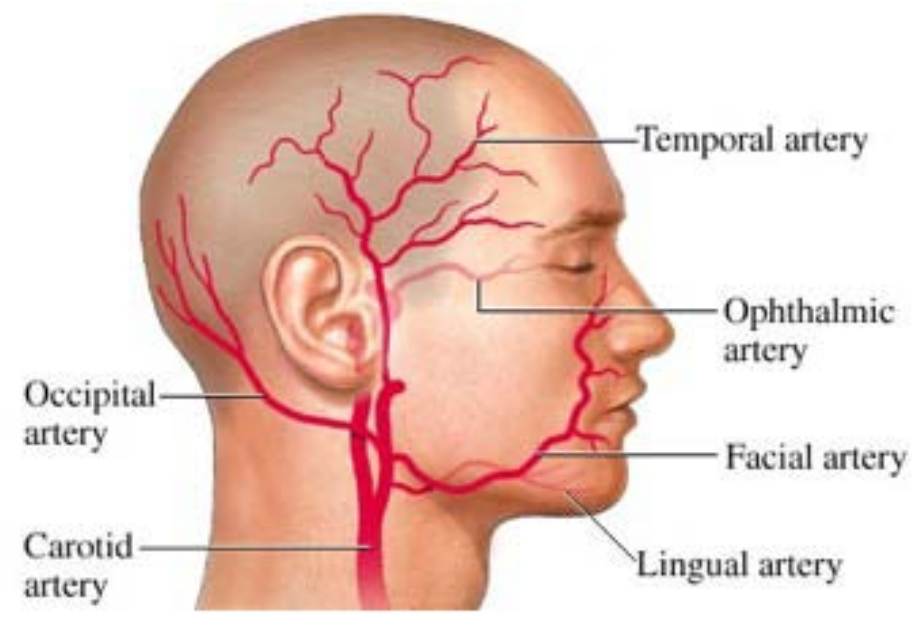

Fig. (1). Arteries commonly affected in GCA.

Table 1. Laboratory Evaluation - Case Report 1

\begin{tabular}{|c|c|c|}
\hline & Before Treatment & 1-Month After Treatment \\
\hline \hline ESR & $100 / 120$ & $5 / 20$ \\
\hline KO $-\mathrm{Hgb}(\mathrm{g} / \mathrm{l})$ & 109 & 0,37 \\
\hline Hct & 0,32 & 117 \\
\hline Wbc $\left(\mathrm{x} 10^{9} / \mathrm{l}\right)$ & 10,3 & 206 \\
\hline Plt $\left(\mathrm{x} 10^{9} / \mathrm{l}\right)$ & 335 & 2,84 \\
\hline Fibrinogen & 6,57 & 0,6 \\
\hline AST (ukat/l) & 1,9 & 0,58 \\
\hline ALT (ukat/l) & 1,56 & 2,0 \\
\hline
\end{tabular}

Three weeks before admission she was examined by general doctor because of headache, weakness, low grade fever and anorexia, simulating a influenza like illness for which supportive treatment was administered. She developed blindness of right eye, while on this treatment.

The consumption of fruit, vegetables and legumes (210 $\mathrm{g}$ /day) were lower and refined carbohydrates; biscuits, cakes, cola drinks and refined bread (955 g/day) were higher than those advised by the International College of Nutrition [10]. Total visible fat $(69 \mathrm{~g} /$ day $)$ intake including trans fatty acids (20g/day), saturated fatty acids (22g/day), linoleic acid ( $15 \mathrm{~g} /$ day) and rich oils (sun flower) were higher, and w-3 fatty acids $(0.46 \mathrm{~g} /$ day $)$ and monounsaturated fat $(12 \mathrm{~g} /$ day $)$ intake was lower than that advised by the health agencies. $\mathrm{W}-6 / \mathrm{W}-3$ ratio(15.0/0.46) of the diet was about 32.6.

Laboratory data showed a highly elevated erythrocyte sedimentation rate (ESR)- over $100 \mathrm{~mm} / \mathrm{h}$, mild hypochromic anemia, elevated fibrinogen, and C-reactive protein. Antinuclear antibodies (ANA, ENA, ACLA, DNP, anti dsDNA) were undetectable. Hepatitis A,B and C were ruled out. Ophthalmic examination confirmed right sides optic nerve ischemic lesions. Brain computerised axial tomography revealed mild cerebral ischemic lesions. (Table 1).

In view of the above diagnosis, she was administered, prednisone $80 \mathrm{mg} /$ day together with vasodilatator therapy.
There was a prompt relief of myalgias, clinical improvement, with reduction in CRP and ESR, but the blidness was irreversible.

\section{CASE REPORT 2}

A 69 year old female, presented with pain and muscle weakness of her shoulders and pelvic girdles, fatigue, anorexia and weight loss. She had a past history of bilateral phlebotrombosis and ischemic heart disease.

Physical examination revealed diffuse muscular pain, tenderness of tendons and joints, muscle wasting and kyphoscoliosis. Her blood pressure was $135 / 80$, and she had a regular pulse rate of 68 per minute. She also had tenderness of her proximal interphalangeal and metacarpophalangeal joints of her hand as well as her elbows, shoulders and pelvic girdle, together with morning stiffness. The symptoms were symmetrical.

The consumption of fruit, vegetables and legumes (217 g/day) were lower, and refined carbohydrates; biscuits, cakes, cola drinks, refined bread (1055 g/day) were higher than those advised by the International College of Nutrition [10]. Total visible fat (66g/day) intake including trans fatty acids (18 g/day), saturated fatty acids (22 g/day), linoleic acid (14g/day) rich oils (sun flower) were also higher and w3 fatty acid $(0.43 \mathrm{~g} /$ day) and monounsaturated fat $(12.0 \mathrm{~g} /$ day $)$ 
intake were lower than those advised by the health agencies(10). The $\mathrm{W}-6 / \mathrm{W}-3$ ratio $(14.0 / 0.43)$ of the diet was about 32.5 .

Laboratory data showed an elevated ESR, anemia, elevated C-reactive protein and fibrinogen. There was no evidence of malignant disease or myositis and patient had a normal EMG findings, and muscles enzymes. She was diagnosis with polymyalgia rheumatica. Treatment with prednosolone $60 \mathrm{mg} /$ day gave rapid recovery in her symptoms. She was discharged from the hospital with relief of her symptoms after the patient herself stopped the treatment. One month after stopping the treatment, the patient was admitted to hospital again, with severe headache, fatigue, ague, and low grade fever. Dilated temporal arteries were present, and the vessels were thickened and tender. Investigation the ESR was greatly elevated and there was a mild hypochromic anemia. The leukocyte and differential counts were normal, C-reactive protein was elevated, and RF was negative. A temporal artery biopsy confirmed the diagnosis of temporal arteritis. Again the treatment with corticosteroid was started with a good clinical response. The patient is now on a low dose of corticosteroid with no symptoms.

\section{DISCUSSION}

This case study shows that $\mathrm{w}-6 / \mathrm{w}-3$ ratio of diets were higher in both patients, one having PMR (32.6) and the other GCA (32.5), compared to ratio advised in the intervention trials $(<5.0)$. The rise in erythrocyte sedimentation rate and elevated C- reactive protein observed in our patients are most important indicators of PMR and GCA, in view of the clinical manifestations in association with normochromic anemia. Although the pathogeneses of GCA and PMR are uncertain, similar cellular immune responses involving $\mathrm{T}$ cells, antigen-presenting cells, macrophage-derived inflammatory cytokines, genetic human leukocyte antigen molecules, and macrophages are found in both conditions [5]. It is possible that the high $\mathrm{w}-6 / \mathrm{w}-3$ ratio of diet (advised nor$\mathrm{mal},<5.0,10)$ and the increased intake of trans fats may have caused a rise in proinflammatory cytokines, resulting in activation of the immune response and manifestion of GCA and PMR [8-13]. There are epidemiological studies and intervention trials available to demonstrate the role of low w6/w-3 ratio diet in the prevention of chronic diseases, particularly cardiovascular diseases [13-20], however such studies are unavailable in relation with GCA and PMR. Our study indicates that such studies are very much needed among these patients [13-20].

The presence of inflammation implies mediation of symptoms by inflammatory lipids and proteins as well as structural damage by proteolytic enzymes. These enzymes can degrade structural proteins within cartilage and synovial membrane of the joint. Polyunsaturated fatty acids (PUFA) of both w- 6 and w-3 classes are essential requirements because we lack the desaturase enzymes, necessary to generate these fatty acids de novo $[17,18]$. Differences in dietary patterns and food choices can generate levels of omega -6 highly unsaturated fatty acids (HUFA), as a proportion of total plasma phospholipid HUFA in free living subjects, that range from 15 to $90 \%$. Most of this omega-6 HUFA is arachidonic acid (AA) and proportions of tissue phospholipids
HUFA correlates closely with plasma phospholipid HUFA. Omega- 6 and omega-3 HUFA are located in the sn2 position of phospholipids, where they compete for incorporation and release and where their levels are related reciprocally. HUFA are released by phospholipase A2 for metabolism by the eicosanoid forming enzymes, such as cyclooxygenases- 1 and$2(\mathrm{COX}-1$ and COX-2) and 5-lipoxygenase. Since the omega3 HUFA eicosapentaenoic acid (EPA) and docosahexaenoic acid (DHA), act as competitive inhibitors of conversion of AA to pro-inflammatory eicosanoids, dietary habits appear to influence propensity for inflammation in our body. This may manifest as low grade persistent vessel wall inflamation in the form of endothelial dysfunction and atherosclerosis or giant cell arteritis. In the case of joints and muscle, inflammation tends to be symptomatic due to their movements resulting into arthritis, arthralgia and myalgia. Several proinflammatory mediators are formed as a result of multistep metabolic processes that involves release of AA $(20: 4$ omega-6) from cell membrane phospholipids. COX acts on AA to form the unstable intermediates which enhance the formation proinflammatory prostaglandins, thrombaxane A2(TX A2) and cytolkines [17, 18].

Dietary omega-3 fats are competitors with homologous omega-6 for remodeling to HUFA and for incorporation into cell membranes [17-19]. EPA (C20:5 omega-3) is the 20 carbon omega-3 homologue of AA and competes with AA for enzymatically mediated release from cell membranes and for metabolism by COX and terminal synthases. AA and EPA are also competitive substrates for 5-LO and down stream enzymes that produce the 5-hydroxy fatty acids and leukotriens which are mediators of inflammation and allergy. The observed effects of omega-3 PUFA on interleukin-1 and TNF-alpha synthesis are important because of the central role of these cytokines play in orchestrating inflammation [17-19]. Their effects include upregulation of expression of proteolytic enzymes that degrade structural proteins in ways that can lead to tissues failure at sites of inflammation.

Polymyalgia rheumatica is clinical syndrome occuring usually in patients over the age of 55 years, characterized by pain and stiffness in the neck, shoulder and pelvic girdles, with variable systemic symptoms and a raised erythrocyte sedimentation rate (ESR). PMR is often associated with gaint cell arteritis (GCA). Up to $40 \%$ of patients with symptoms of PMR have arteritis in temporal artery biopsy specimens [1]. Inflammatory arthritis occurs in some cases of PMR and GCA [2]. The musculoskeletal symptoms are usually bilateral and symmetrical. Stiffness is usually predominant feature, and it is severe after the rest. Muscular pain is diffuse and is common at night [3, 4]. Arterial biopsies often reveal inflammatory changes, which cause narrowing or occlusion of the vessel leading to ischemia distal to the lesion. GCA most commonly involves the branches of the internal and external carotid arteries. The involvement of these branches leads to the clinical findings of headache, scalp tenderness and blindness [5, 7]. Biopsy of the temporal artery is very important in the diagnosis of GCA which was not done in our cases.

In one study [20], the therapeutic efficacy and tolerability of intravenously applied n-3-PUFA in patients with active rheumatoid arthritis (RA) was examined. Thirty-four patients with active RA [identified as having a DAS28 (disease activ- 
ity score including a 28 joint count) $>4$.0] were enrolled into this 5-wk open pilot study (one group design). From the time of screening (visit 0 , or V0), background therapy had to remain unchanged. Patients received $2 \mathrm{~mL} / \mathrm{kg}(=0.1-0.2 \mathrm{~g}$ fish oil $/ \mathrm{kg}$ ) fish oil emulsion intravenously on 7 consecutive days (Visit 1-Visit 2, or V1-V2) in addition to their background therapy. A decrease of the DAS28 $>0.6$ at day 8 (Visit 2) was the primary efficacy measure. Moreover, the DAS28 at day 35 (Visit 3, or V3), the modified Health Assessment Questionnaire, the American College of Rheumatology (ACR) response criteria (V2, V3) and the Short Form-36 (V3) were assessed. Thirty-three patients completed the trial. The mean DAS28 at V1 was 5.45; at V2, 4.51 ( $<<.001$ V1$\mathrm{V} 2)$ and at V3, $4.73(\mathrm{P}<.001 \mathrm{~V} 1-\mathrm{V} 3$; V2-V3, not significantly different). Of the 34 patients, $56 \%$ achieved a reduction of the DAS28 $>0.6$ at V2 (mean 1.52); 27\% $>1.2$. At $\mathrm{V} 3,41 \%$ of the patients showed a DAS28 reduction $>0.6$ (mean 1.06), and $36 \%>1.2$. ACR 20 and 50\% responses at V2 were seen in 29 and $12 \%$ of patients, respectively; at V3, the comparable values were 18 and $9 \%$, respectively. Overall tolerability was excellent. Intravenous application of n-3PUFA (as an add-on therapy) was considerably well tolerated and led to improvement of the disease activity status in a reasonable number of RA patients. Future trials are warranted to answer whether the intravenous application of n-3PUFA constitutes a therapeutic option in RA patients.

Low dosage prednisolone (10-20 $\mathrm{mg}$ ) improves the symptoms of PMR. The duration of therapy commonly is two to three year on a lower dosage of prednisolone. Corticosteroids are the first-line therapy also for GCA, but in higher dosage. Our patients were treated according to an accepted therapeutic regiment with corticosteroids [6]. Dramatic improvement of clinical and laboratory findings occured. Visual disturbances - visual loss in the right eye (case report 1) was irreversible. It is possible that due to aggressive treatment, involvement of the second eye did not occur. Polymyalgia rheumatica and giant cell arteritis may be an ophtalmic emergency, because they may cause loss of vision. Early diagnosis and treatment is the precondition for preserving the patient's eye sight. Prevention of both the diseases may be possible by increasing the intake of $w-3$ fatty acids and by decreasing intake of $w-6$ fatty acids to have a diet with a lower $w-6 / w-3$ ratio $<5.0$. Omega-3 fatty acids can decrease proinflammatory cytokines and reverse the immune response which may be protective against recurrence of GCA and PMR among these patients. Omega-3 fatty acids are rich in fish, fish oil, walnuts, flex seeds, chia seeds, mustered oil and canola oil which may be preferred in the diet for adequate balance of these fatty acids.

In brief, our findings indicate that role of w-3 fatty acids needs further study in the prevention and treatment of PMR and GCA.

\section{REFERENCES}

[1] Behn AR, Perera T, Myles AB. Polymyalgia rheumatica and corticosteroids: how much for how long? Ann Rheum Dis 1983; 42: 374-8.

[2] O Duffy JD, Hunder GG, Wahner HW. A follow-up study of polymyalgia rheumatica: evidence of chronic axial synovitis. J Rheumatol 1980; 7: 685-93.

[3] Bird HA, Leeb BF, Montecucco CM, et al. A comparision of sensitivity of diagnostic criteria for polymyalgia rheumatica. Ann Rheum Dis 2005; 64: 626-9.

[4] Langford CA. Vasculitis in the geriatric population. Clin Geriatr Med 2005; 21: 631-47.

[5] Weyand CM, Goronzy JJ. Medium and large-vessel vasculitis. N Engl J Med 2003; 349: 160-9.

[6] Zimmermann-Gorska I. Polymyalgia rheumatica: clinical picture and principles of treatment. Pol Arch Med Wewn 2008; 118: 377-80.

[7] Schmidt D. Ocular ischemia syndrome - malignant course of giant cell arteritis. Eur J Med Res 2005; 10: 233-42.

[8] Ailhaud G, Massiéra F, Weill P, Legrand P, Alessandri JM, Guesnet $\mathrm{P}$. Temporal changes in dietary fats: role of $n-6$ polyunsaturated fatty acids in excessive adipose tissue development and relationship to obesity. Prog Lipid Res 2006; 45: 203-36.

[9] Endres S, Ghorbani R, Kelley VE. The effect of dietary supplementation with $n-3$ polyunsaturated fatty acids on the synthesis of interleukin-1 and tumor necrosis factor by mononuclear cells. N Engl J Med 1989; 320: 265-68.

[10] Singh RB, De Meester F, Pella D, Basu TK, Watson R. Globalization of dietary wild foods protect against cardiovascular disease and all cause mortalities? A Scientific satement from the International College of Cardiology, Columbus Paradigm Institute and the International College of Nutrition. Open Nutra J 2009; 2: 42-45.

[11] Simopoulos AP. Is insulin resistance influenced by dietary linoleic acid and trans fatty acids? Free Radic Biol Med 1994; 17: 367-72.

[12] Appel LJ. Dietary patterns and longevity. Circulation 2008; 118 : 214-15.

[13] Singh RB, DeMeester F, Mechirova V, Pella D, Otsuka K. Fatty acids in the causation and therapy of metabolic syndrome. In: DeMeester F, Watson RR, Eds. Wild type foods in health promotion and disease prevention, Humana Press, NJ 2008; p. 26

[14] Singh RB, Dubnov G, Niaz MA, et al. Effect of an IndoMediterranean diet on progression of coronary disease in high risk patients: a randomized single blind trial. Lancet 2002; 360: 145561.

[15] Singh RB, Rastogi SS, Verma R, Bolaki L, Singh R. An Indian experiment with nutritional modulation in acute myocardial infarction. Am J Cardiol 1992; 69: 879-85.

[16] Heidemann C, Schulze MB, Franco OH, van Dam RM, Mantzoros CS, Hu FB. Dietary patterns and risk of mortality from cardiovascular disease, cancer, and all causes in a prospective cohort of women. Circulation 2008; 118: 230-7.

[17] Lands WEM, Libelt B, Morris A, et al. Maintenance of lower proportions of $n-6$ eicosanoids precursors in phospholipids of human plasma in response to added dietary n-3 fatty acids. Biochem Biophys Acta 1992; 1180: 147-62.

[18] Harvald B. Genetic epidemiology of greenland. Clin Genet 1989; 36: 364-7.

[19] De Meester F. Progress in lipid nutrition: the Columbus Concept addressing chronic diseases. In: Simopoulos AP, De Meester F, Eds. A balanced omega-6/omega-3 fatty acid ratio, cholesterol and coronary heart disease. World Review of Nutrition and Dietetics, Simopoulos AP, series ed. Basel: Karger 2009; 100: pp. 110-121.

[20] Leeb BF, Sautner J, Andel I, Rintelen B. Intravenous application of omega-3 fatty acids in patients with active rheumatoid arthritis. The ORA-1 trial. An open pilot study. Lipids 2006; 41: 29-34. 